\title{
Anomalous dimensions of operators without derivatives in the non-linear $\sigma$-model for disordered bipartite lattices
}

\author{
Luca Dell'Anna \\ Max-Planck-Institut für Festkörperforschung, D-70569 Stuttgart, Germany and \\ Institut für Theoretische Physik, Heinrich-Heine-Universität, D-40225 Düsseldorf, Germany.
}

\begin{abstract}
We consider a generic time-reversal invariant model of fermions hopping randomly on a square lattice. By means of the conventional replica-trick within the fermionic path-integral formalism, the model is mapped onto a non-linear $\sigma$-model with fields spanning the coset $U(4 N) / S p(2 N), N \rightarrow 0$. We determine the proper scaling combinations of an infinite family of relevant operators which control deviations from perfect two-sublattice symmetry. This allows us to extract the low-energy behavior of the density of states, which agrees with earlier results obtained in particular two-sublattice models with Dirac-like single-particle dispersion. The agreement proves the efficacy of the conventional fermionic-path-integral approach to disordered systems, which, in spite of many controversial aspects, like the zero-replica limit, remains one of the more versatile theoretical tool to deal with disordered electrons.
\end{abstract}

PACS numbers: 73.20.Jc; 73.20.Fz; 71.30.+h

\section{INTRODUCTION}

It is known that localization does not occur in any dimension at the band-center energy of tight binding models on bipartite lattice-Hamiltonians [1, 2, 3, 4, 5, 6, 7, 8, 9, 10, 11]. Gade and Wegner 4, 5] first realized that these models correspond to a particular class of non-linear $\sigma$-models in the zero replica limit, so called two-sublattice models, which exhibit an additional chiral symmetry 4, , 5, 8]. As a consequence of this symmetry, they were able to prove that, when the chemical potential is right in the centre of the band, quantuminterference corrections to the $\beta$-function vanish exactly. In addition they showed that, unlike conventional disordered systems, the density of states (DOS) $\rho(E)$ near the bandcenter $E=0$ is strongly affected by disorder. They actually predicted a diverging behavior 
[4, 5] (see also Refs. [8, [9])

$$
\rho(E) \sim \frac{1}{|E|} \mathrm{e}^{-A \sqrt{\ln |B / E|}}
$$

where $A$ and $B$ are positive constants.

The subleading dependence $\exp (-A \sqrt{\ln |B / E|})$ has been recently questioned by Motrunich, Damle and Huse [12]. They analysed the strong disorder limit of a set of models which belongs to the two-sublattice class and found that the correct subleading dependence is instead $\exp \left(-A|\ln B / E|^{2 / 3}\right)$. This result was later confirmed by field-theoretical approaches based on supersymmetry [13] and on replica trick [14] applied to the so-called Hatsugai-Wen-Kohmoto (HWK) model, which describes electrons hopping randomly on a square lattice in the presence of a $\pi$-flux per plaquette. This model is particularly suitable for a weak-disorder field-theoretical approach. Indeed, for uniform hopping, the low-energy single-particle spectrum of the HWK model is composed by two Dirac-like cones, which allows the use of the full machinery of Conformal Field Theory when a weak random-hopping component is included. The important breakthrough put forward by these analyses is that many disorder-average quantities, like the density of states, are determined by an infinite set of relevant local operators with negative dimensions [13].

In reality, these new results raise an intriguing question about the concept of universality commonly accepted in disordered systems, according to which the HWK model should be representative of any two-sublattice model since the action of its long-wavelength diffusive modes is a non-linear $\sigma$-model in the same universality class as generic two-sublattice models. However the results found by Mudry, Ryu, and Furusaki [13] and by Yamada and Fukui [14] have been obtained working directly with the HWK Hamiltonian without going through the non-linear $\sigma$-model mapping, just thanks to the Dirac-like dispersion. Lacking an independent derivation starting from the non-linear $\sigma$-model, it is not a priori obvious to what extent these results are actually generic to any two-sublattice model.

In this work, we are going to show that the results of Refs. [12, 13, 14] can be fully recovered through the conventional non-linear $\sigma$-model approach based on the replica-trick within the fermionic path-integral formalism, without assuming any Dirac-like dispersion. Besides satisfying a purely theoretical curiosity, this result proves once again the strength of the conventional approach to disordered systems.

The paper is organized as follows. In Section II we introduce the model as well as the nonlinear $\sigma$-model which describes its long-wavelength diffuse modes. In Section III we analyse 
the scaling behavior of some operators which are compositions of such diffuse modes, called soft operators, and whose $\beta$-functions are solved in Section IV. Finally in Section V we apply what found in the previous sections to calculate the mean density of states near the center of the band.

\section{THE MODEL}

A two-sublattice model is described by a non-interacting Hamiltonian on a bipartite lattice, with sublattices $A$ and $B$, of the general form

$$
\mathcal{H}=-\sum_{R \in A} \sum_{R^{\prime} \in B} t_{R R^{\prime}} c_{R}^{\dagger} c_{R^{\prime}}+H . c .,
$$

with random matrix elements $t_{R R^{\prime}}$ which only connect one sublattice to the other. It follows that, if $\Psi(R)$ is an eigenstate with eigenvalue $E$, then the wavefunction $\phi(R) \Psi(R)$, where $\phi(R)=1$ if $R \in A$ and $\phi(R)=-1$ if $R \in B$, is also an eigenstate with eigenvalue $-E$. This also implies that any eigenstate at $E=0$ is doubly degenerate, unless boundary conditions break the degeneracy between the two sublattices. Following Ref. [15], we introduce, within the fermionic path-integral formalism, the following Nambu spinors

$$
\psi_{R}=\frac{1}{\sqrt{2}}\left(\begin{array}{l}
\bar{c}_{R} \\
c_{R}
\end{array}\right)
$$

where $c_{R}$ and $\bar{c}_{R}$ are Grassmann variables with components $c_{R, p, a}$ and $\bar{c}_{R, p, a}$ in which $R$ refers to a lattice site, $p= \pm$ is the index of positive or negative frequency components, $a=1, . ., N$ is the replica index which has to be sent to zero at the end of the calculation. In addition we define conjugate fields through

$$
\bar{\psi}_{R}=\left[\hat{c} \psi_{R}\right]^{t}=\frac{1}{\sqrt{2}}\left(-c_{R}, \bar{c}_{R}\right),
$$

where $\hat{c}$ is the charge conjugation matrix $\hat{c}=-i \tau_{2}$. Here and what follows the $\tau_{i}$ 's, $i=1, \ldots, 3$, are Pauli matrices acting in the Nambu space. With these fields the action describing the model at a fixed disorder configuration for fields with energy $E \pm i \omega$ is

$$
S=-\sum_{R \in A} \sum_{R^{\prime} \in B} \bar{\psi}_{R}\left(E \delta_{R R^{\prime}}-i \omega s_{3} \delta_{R R^{\prime}}-t_{R R^{\prime}}\right) \psi_{R},
$$


where $s_{3}$ is the third Pauli matrix acting on the two frequency components $E \pm i \omega$ and the frequency transferred $2 \omega$ plays the role of a symmetry breaking field. Since the detailed derivation of the non-linear $\sigma$-model starting from the action (2) is known, see for instance Ref. [8], here we just outline the main steps emphasizing the peculiarity of two-sublattice models. We start noticing that the action when $E=\omega=0$ is invariant under the transformation

$$
\psi_{R} \rightarrow \mathrm{e}^{i \alpha \phi(R)} \psi_{R}, \quad \bar{\psi}_{R} \rightarrow \bar{\psi}_{R} \mathrm{e}^{i \alpha \phi(R)}
$$

which is allowed within the path-integral formalism since the Grassmann variables $c_{R}$ and $\bar{c}_{R}$ are independent. This additional abelian symmetry plays an important role in these models, as originally recognized by Gade and Wegner 4]. Within the replica-trick technique, the average of disorder can be performed directly on the action (21) and generates a non-local interaction which connects two sites belonging to different sublattices. This interaction is then decoupled by an Hubbard-Stratonovich transformation, introducing an auxiliary field $Q$. Due to the non-locality of the interaction, in the long-wavelength limit this field has two components, one uniform, $Q_{0}(R)$, and the other staggered, $\phi(R) Q_{3}(R)$, where both $Q_{0}$ and $Q_{3}$ vary smoothly in space. In particular, if we introduce the Pauli matrices $\gamma_{i}$, $i=1,2,3$, as well as the identity matrix $\gamma_{0}$ in the two-sublattice space, the action of $\gamma_{3}$ being just that of $\phi(R)$, the auxiliary field to which the electron density is coupled is $Q(R)=Q_{0}(R) \gamma_{0}+i Q_{3}(R) \gamma_{3}$. Both $Q_{0}$ and $Q_{3}$ are $4 N \times 4 N$ hermitian matrices in Nambu, energy and replica spaces $[4 N=2(\mathrm{Nambu}$ spinor dimension $) \times 2$ (frequency components) $\times N$ (replicas)]. The derivation proceeds then through the following steps: i) integrating over the Grassmann variables, ii) expanding the effective action around the symmetry breaking saddle point $Q_{0}=\Sigma s_{3}$, where $\Sigma$ is the inverse relaxation time, iii) integrating out massive modes and just focusing on low-energy long-wavelength transverse fluctuations. In this way one obtains the following effective action for the auxiliary field when $E=0$ [5, 8$]$,

$$
F_{0}=\int d r\left[\frac{\pi \sigma}{4^{2}} \operatorname{Tr}\left(\nabla Q^{\dagger}(r) \nabla Q(r)\right)-\frac{\pi \Pi}{2 \cdot 4^{3}}\left(\operatorname{Tr}\left(Q^{\dagger}(r) \nabla Q(r) \gamma_{3}\right)\right)^{2}-\frac{\pi \nu \omega}{4} \operatorname{Tr}\left(s_{3} Q(r)\right)\right]
$$

In the above equation we have rescaled $Q \rightarrow \Sigma Q$ with $Q=\widetilde{U}^{\dagger} s_{3} U$ and the unitary transformation $U \in U(4 N) / S p(2 N)$ fulfilling

$$
\widetilde{U}^{\dagger} \equiv \gamma_{1} U^{\dagger} \gamma_{1}=\hat{c} U^{t} \hat{c}^{t}
$$


The latter relation implies the constrain $Q^{\dagger} Q=1$. The coupling $\sigma$ is the conductivity in Born approximation, $\Pi$ is related to the staggered density of states fluctuations [8] and $\nu$ is the density of states at the chemical potential. The transformation $U$ can be written as $U=e^{W}$ with $W=W^{0} \gamma_{0}+W^{3} \gamma_{3}$. The charge conjugation invariance implies for the $Q$-fields the following relation

$$
\hat{c} Q^{t} \hat{c}^{t}=Q \text {. }
$$

\section{A. Gaussian propagators}

In the Wilson-Polyakov renormalization group (RG) approach [16] one assumes a separable form for the transformation $U=U_{(f)} U_{(s)}$ where $U_{(f)}$ involves fast modes with momentum $q \in[\Lambda / s, \Lambda]$, while $U_{(s)}$ involves slow modes with momentum $q \in[0, \Lambda / s]$, with $\Lambda$ being the high momentum cut-off, and $s>1$ the rescaling factor. By introducing upper indices, $a, b, c, \ldots$, in Nambu space which assume the values 1 and 2 , and lower multindices in replica and energy spaces $(n=(r, p)$, with $r$ being the replica index and $p$ the sign of the frequency) we expand $Q$ to the second order in $W$-fast obtaining

$$
Q_{n_{1} n_{2}}^{a d} \simeq Q_{n_{1} n_{2}(s)}^{a d}+\widetilde{U}_{n_{1} m_{1}}^{\dagger a b} S_{m_{1}} W_{m_{1} m_{2}}^{b c} U_{m_{2} n_{2}}^{c d}+\frac{1}{2} \widetilde{U}_{n_{1} m_{1}}^{\dagger a b} S_{m_{1}} W_{m_{1} l}^{b c^{\prime}} W_{l m_{2}}^{c^{\prime} c} U_{m_{2} n_{2}}^{c d} .
$$

In the above equation the sums over repeated indices are assumed, the label $(s)$ is dropped in $U$ and $S_{n}=p$ is the sign of the infinitely small frequency related to the index $n$. The gaussian propagator reads

$$
\begin{array}{r}
\left\langle W_{n m}^{\alpha, a b}(k) W_{l p}^{\alpha, c d}(k)\right\rangle=\left((-)^{\alpha} S_{n} S_{m}-1\right) D^{\alpha}(k)\left\{\delta ^ { a 1 } \delta ^ { b 1 } \left(\delta^{c 1} \delta^{d 1} \delta_{n p} \delta_{m l}\right.\right. \\
\left.-(-)^{\alpha} \delta^{c 2} \delta^{d 2} \delta_{n l} \delta_{m p}\right)+\left(\delta^{a 1} \delta^{b 2} \delta^{c 2} \delta^{d 1}+\delta^{a 2} \delta^{b 1} \delta^{c 1} \delta^{d 2}\right) \\
\left(\delta_{n p} \delta_{m l}+(-)^{\alpha} \delta_{n l} \delta_{m p}\right)+\delta^{a 2} \delta^{b 2}\left(\delta^{c 2} \delta^{d 2} \delta_{n p} \delta_{m l}-(-)^{\alpha} \delta^{c 1} \delta^{d 1} \delta_{n l} \delta_{m p}\right) \\
\left.+\Gamma \delta_{\alpha 3} \delta^{a b} \delta^{c d} \delta_{n m} \delta_{l p} / 2\right\},
\end{array}
$$

where $\alpha=0,3$ and

$$
\begin{aligned}
& D^{0}(k)=D^{3}(k)=\frac{1}{\pi \sigma k^{2}}, \\
& \Gamma=\frac{\Pi}{\sigma+N \Pi},
\end{aligned}
$$

with $N$ are the number of replicas that we send to zero. The relation in Eq. (44) implies

$$
U_{n m}^{a b}=\left(\delta^{a b}-1\right) \widetilde{U}_{m n}^{\dagger a b}+\delta^{a b} \widetilde{U}_{m n}^{\dagger a+1 b+1}=(-)^{a+b} \widetilde{U}_{m n}^{\dagger b+1 a+1},
$$


with the clock rules $1+1=2$ and $2+1=1$ for the upper indices. From Eq. (77) and Eq. (10) we obtain

$$
\begin{aligned}
& \left\langle Q_{n_{1} n_{2}}^{a b}\right\rangle=Q_{n_{1} n_{2}}^{a b}+L_{3} \frac{1}{2}(2-8 N+\Gamma) Q_{n_{1} n_{2}}^{a b}, \\
& \left\langle Q_{n_{1} n_{2}}^{a b} Q_{n_{3} n_{4}}^{c d}\right\rangle=Q_{n_{1} n_{2}}^{a b} Q_{n_{3} n_{4}}^{c d}+L_{0}\left[(-)^{b+c} Q_{n_{1} n_{3}}^{a c+1} Q_{n_{2} n_{4}}^{b+1 d}-Q_{n_{1} n_{4}}^{a d} Q_{n_{3} n_{2}}^{c b}\right] \\
& +L_{3}\left[(2-8 N+2 \Gamma) Q_{n_{1} n_{2}}^{a b} Q_{n_{3} n_{4}}^{c d}+(-)^{b+c} Q_{n_{1} n_{3}}^{a c+1} Q_{n_{2} n_{4}}^{b+1 d}-Q_{n_{1} n_{4}}^{a d} Q_{n_{3} n_{2}}^{c b}\right]
\end{aligned}
$$

where $L_{\alpha}=\int_{\Lambda / s}^{\Lambda} \frac{d \vec{k}}{(2 \pi)^{d}} D^{\alpha}(k)$ and the label $(s)$ is dropped in all the $Q$-fields on the right-hand side of the equations. Eq. (5) yields the properties

$$
\begin{aligned}
& Q_{n m}^{11}=Q_{m n}^{22} \\
& Q_{n m}^{12}=-Q_{m n}^{12}
\end{aligned}
$$

which imply some further properties, for instance,

$$
\sum_{a c}(-)^{a+c} Q_{n_{1} n_{3}}^{a c+1} Q_{n_{2} n_{4}}^{a+1 c}=-\sum_{a c} Q_{n_{1} n_{3}}^{a c} Q_{n_{4} n_{2}}^{c a}
$$

useful to evaluate product of traces. From Eqs. (12) and (15), we get

$$
\begin{aligned}
\left\langle\sum_{a b} Q_{n_{1} n_{2}}^{a a} Q_{n_{3} n_{4}}^{b b}\right\rangle & \left.\left.=\sum_{a b}\left\{[1+2 L(1+\Gamma)] Q_{n_{1} n_{2}}^{a a} Q_{n_{3} n_{4}}^{b b}-2 L\left[Q_{n_{1} n_{3}}^{a b} Q_{n_{4} n_{2}}^{b a}+Q_{n_{1} n_{4}}^{a b} Q_{n_{3} n_{2}}^{b a}\right]\right\},(16)\right] Q_{n_{1} n_{4}}^{a b} Q_{n_{3} n_{2}}^{b a}+2 L\left[Q_{n_{1} n_{3}}^{a b} Q_{n_{4} n_{2}}^{b a}-Q_{n_{1} n_{2}}^{a a} Q_{n_{3} n_{4}}^{b b}\right]\right\},(1 \\
\left\langle\sum_{a b} Q_{n_{1} n_{4}}^{a b} Q_{n_{3} n_{2}}^{b a}\right\rangle & =\sum_{a b}^{a b}\left\{[1+2 L(1+\Gamma)] Q_{n_{1} n_{3}}^{a b} Q_{n_{4} n_{2}}^{b a}+2 L\left[Q_{n_{1} n_{4}}^{a b} Q_{n_{3} n_{2}}^{b a}-Q_{n_{1} n_{2}}^{a a} Q_{n_{3} n_{4}}^{b b}\right]\right\}, \\
\left\langle\sum_{a b} Q_{n_{1} n_{3}}^{a b} Q_{n_{4} n_{2}}^{b a}\right\rangle & =\sum_{a b}\{[1+2 L(1+\Gamma
\end{aligned}
$$

where $N=0$ and $L=L_{0}=L_{3}$. From the equations above we notice that the oneloop calculation leads to transpositions of the indices under the sum over Nambu space and that Eqs. (16, 18) form a closed set of equations. Defining $v_{1}=\sum_{a b} Q_{n_{1} n_{2}}^{a a} Q_{n_{3} n_{4}}^{b b}$, $v_{2}=-\sum_{a b} Q_{n_{1} n_{4}}^{a b} Q_{n_{3} n_{2}}^{b a}$ and $v_{3}=-\sum_{a b} Q_{n_{1} n_{4}}^{a b} Q_{n_{3} n_{2}}^{b a}$, the equations above can be summarized by

$$
\left\langle v_{i}\right\rangle=v_{i}+2 L \Gamma v_{i}+2 L\left(v_{1}+v_{2}+v_{3}\right)
$$

Applying a rotation we can obtain three independent scaling operators $\widetilde{v}_{1}=v_{1}+v_{2}+v_{3}$, $\widetilde{v}_{2}=v_{1}-v_{2}$ and $\widetilde{v}_{3}=v_{2}-v_{3}$, with the following scaling behaviors

$$
\begin{aligned}
& \left\langle\widetilde{v}_{1}\right\rangle=\widetilde{v}_{1}+2 L(3+\Gamma) \widetilde{v}_{1}, \\
& \left\langle\widetilde{v}_{i}\right\rangle=\widetilde{v}_{i}+2 L \Gamma \widetilde{v}_{i}, \quad \text { for } i=2,3 .
\end{aligned}
$$


From Eqs. (11) and (12) one can evaluate the scaling behavior at one loop level of all the composite operators for the model (3) with symmetry $U(4 N) / S p(2 N)$. Another result which can be easily obtained from Eqs. (111) and (12) is the mean value of the full product of $n$ matrices $Q$ in the limit $N \rightarrow 0$

$$
\left\langle Q^{n}\right\rangle=Q^{n}+L\left[\frac{n^{2}}{2}(2+\Gamma) Q^{n}-n \sum_{l=1}^{n-1} Q^{n-l} \operatorname{Tr}^{\prime} Q^{l}\right],
$$

where $\operatorname{Tr}^{\prime}$ is a trace which does not act on the sublattice space and $\Gamma=\Pi / \sigma$.

In the absence of the sublattice (or chiral) symmetry, the transverse modes take values in the manifold $S p(2 N) / S p(N) \times S p(N)$. In such a case the gaussian propagator (7) is valid only for $\alpha=0$, implying that $\left\langle Q_{n_{1} n_{2}}^{a b}\right\rangle=Q_{n_{1} n_{2}}^{a b}$ and

$$
\left\langle Q_{n_{1} n_{2}}^{a b} Q_{n_{3} n_{4}}^{c d}\right\rangle=Q_{n_{1} n_{2}}^{a b} Q_{n_{3} n_{4}}^{c d}+L_{0}\left[(-)^{b+c} Q_{n_{1} n_{3}}^{a c+1} Q_{n_{2} n_{4}}^{b+1 d}-Q_{n_{1} n_{4}}^{a d} Q_{n_{3} n_{2}}^{c b}\right],
$$

while Eq. (22) is replaced by

$$
\left\langle Q^{n}\right\rangle=Q^{n}+L\left[\frac{n}{2}(n-1)\right] Q^{n}
$$

\section{SOFT OPERATORS}

Let us consider now the following linear combination of moments of $Q$

$$
P_{n}=\sum_{\mathcal{N}=1}^{n} \sum_{\left\{n_{i}\right\}_{\mathcal{N}}} \lambda_{\left(\left\{n_{i}\right\}_{\mathcal{N}}\right)}^{(\mathcal{N})} \prod_{i=1}^{\mathcal{N}} \operatorname{Tr}^{\prime} Q^{n_{i}}(r),
$$

where $\forall \mathcal{N} \in\{1, \ldots, n\}$, all the sets of $\mathcal{N}$ positive ordered integers $\left\{n_{i}\right\}_{\mathcal{N}}=\left\{n_{1}, \ldots, n_{\mathcal{N}}\right\}$ are such that $\sum_{i=1}^{\mathcal{N}} n_{i}=n$, namely $\left\{n_{i}\right\}_{\mathcal{N}}$ is a partition of $n$ in $\mathcal{N}$ terms. The coefficients $\lambda_{\left(\left\{n_{i}\right\}_{\mathcal{N}}\right)}^{(\mathcal{N})}$ are symmetric with respect to any transpositions of the indices, $\lambda_{\left(n_{1}, \ldots, n_{j}, \ldots, n_{i}, \ldots, n_{\mathcal{N}}\right)}^{(\mathcal{N})}=$ $\lambda_{\left(n_{1}, \ldots, n_{i}, \ldots, n_{j}, \ldots, n_{\mathcal{N}}\right)}^{(\mathcal{N})}$. The number of couplings is given by the number of partitions of $n$, $\sum_{\mathcal{N}=1}^{n} \sum_{\left\{n_{i}\right\}_{\mathcal{N}}} 1=p_{n}$. The trace $\operatorname{Tr}^{\prime}$ is over all the degrees of freedom except those of the sublattice space in order not to miss operators induced by the renormalization. In this way, as we will see below, we have in Eq. (25) a complete set of operators transforming one to the other under the action of the renormalization group. 
From Eqs. (11) and (12) we get the following scaling behavior

$$
\begin{aligned}
& \left\langle\prod_{i=1}^{\mathcal{N}} \operatorname{Tr}^{\prime} Q^{n_{i}}\right\rangle=\left[1+L\left(\sum_{i=1}^{\mathcal{N}} n_{i}^{2}+n^{2} \frac{\Gamma}{2}\right)\right] \prod_{i=1}^{\mathcal{N}} \operatorname{Tr}^{\prime} Q^{n_{i}} \\
& -L\left[4 \sum_{i>j}\left(n_{i} n_{j} \operatorname{Tr}^{\prime} Q^{n_{i}+n_{j}} \prod_{k \neq i, j}^{\mathcal{N}} \operatorname{Tr}^{\prime} Q^{n_{k}}\right)+\sum_{i=1}^{\mathcal{N}}\left(n_{i} \prod_{j \neq i}^{\mathcal{N}} \operatorname{Tr}^{\prime} Q^{n_{j}} \sum_{p=1}^{n_{i}-1} \operatorname{Tr}^{\prime} Q^{n_{i}-p} \operatorname{Tr}^{\prime} Q^{p}\right)\right]
\end{aligned}
$$

for a generic product of traces of any power of $Q$. In the latter equation $\sum_{i>j}$ is a double sum and represents $\sum_{i=1}^{\mathcal{N}} \sum_{j=1}^{i-1}$. In the following we give two particular examples: i) For $\mathcal{N}=1, n_{1}=n$, using Eq. (26), we get

$$
\left\langle\operatorname{Tr}^{\prime} Q^{n}\right\rangle=\left[1+L\left(n^{2}+n^{2} \frac{\Gamma}{2}\right)\right] \operatorname{Tr}^{\prime} Q^{n}-L n \sum_{p=1}^{n-1} \operatorname{Tr}^{\prime} Q^{n-p} \operatorname{Tr}^{\prime} Q^{p}
$$

ii) For $\mathcal{N}=n$, then $\forall i n_{i}=1$, and we obtain in this case

$$
\left\langle\left(\operatorname{Tr}^{\prime} Q\right)^{n}\right\rangle=\left[1+L\left(n+n^{2} \frac{\Gamma}{2}\right)\right]\left(\operatorname{Tr}^{\prime} Q\right)^{n}-2 L n(n-1) \operatorname{Tr}^{\prime} Q^{2}\left(\operatorname{Tr}^{\prime} Q\right)^{n-2}
$$

In the first term of Eq. (26) the product of the $\mathcal{N}$ traces is reproduced, in the second term the number of traces instead is decreased by one, it is $\mathcal{N}-1$, while in the third term the number of traces is increased by one, $\mathcal{N}+1$. In any case the sum of the exponents of $Q$ is equal to $n$. We can say, therefore, that for each positive integer $n$, the set of all the equations (26), with all positive integers $\mathcal{N}$ and $\left\{n_{i}\right\}$ such that $\sum_{i=1}^{\mathcal{N}} n_{i}=n$, is a closed set of RG equations whose number is equal to the number $p_{n}$ of partitions of $n$.

The corresponding $p_{n} \beta$-functions for the couplings, $d \lambda_{\left(\left\{n_{i}\right\}_{\mathcal{N}}\right)}^{(\mathcal{N})} / d \ln s$, are the following

$$
\begin{aligned}
& \beta_{\lambda_{\left(n_{1}, ., n_{\mathcal{N}}\right)}^{(\mathcal{N})}}=d \lambda_{\left(n_{1}, . ., n_{\mathcal{N}}\right)}^{(\mathcal{N})}+g\left[\left(\sum_{i=1}^{\mathcal{N}} n_{i}^{2}+n^{2} \frac{\Gamma}{2}\right) \lambda_{\left(n_{1}, ., n_{\mathcal{N}}\right)}^{(\mathcal{N})}\right. \\
& -4\left(1-\delta_{\mathcal{N}, n}\right) \sum_{i=1}^{\mathcal{N}} \sum_{\bar{n}=1}^{n_{i}-1} K_{\bar{n}, n_{i}-\bar{n}}^{(\mathcal{N}+1)} \lambda_{\left(n_{1}, ., n_{j}, ., n_{i}-\bar{n}_{, ., n}, n_{\mathcal{N}}, \bar{n}\right)}^{(\mathcal{N}+1)} \bar{n}\left(n_{i}-\bar{n}\right) \\
& -\left(1-\delta_{\mathcal{N}, 1)} \sum_{i=1}^{\mathcal{N}} \sum_{j=1}^{i-1} \frac{1}{K_{n_{i}, n_{j}}^{(\mathcal{N})}} \lambda_{\left(n_{1}, ., n_{j}, ., n_{i}+n_{j}, ., n_{\mathcal{N}}\right)}^{(\mathcal{N}-1)}\left(n_{i}+n_{j}\right)\right],
\end{aligned}
$$

where $n_{1} \geq n_{2} \geq \ldots \geq n_{\mathcal{N}}$ and $g=\frac{1}{2 \pi^{2} \sigma}$ is the resistivity. In Eq. (27) the factor $K_{n_{i}, n_{j}}^{(\mathcal{N})}$ is defined by

$$
K_{n_{i}, n_{j}}^{(\mathcal{N})}=\frac{m_{n_{i}}^{(\mathcal{N})}\left(m_{n_{j}}^{(\mathcal{N})}-\delta_{n_{i} n_{j}}\right)}{2 m_{n_{i}+n_{j}}^{(\mathcal{N}-1)}}
$$


where $m_{n_{i}}^{(\mathcal{N})}$ is the number of times the value of the integer $n_{i}$ is repeated in the string $\left(n_{1}, . ., n_{\mathcal{N}}\right)$ or equivalently, $m_{n_{i}}^{(\mathcal{N})}=\left(l_{M}-l_{m}\right)$ where $l_{M}>l_{m} \in \mathbb{N}$ such that $i \in\left\{l_{M}+1, \ldots, l_{m}\right\}$ and $\forall l \in\left\{l_{M}+1, \ldots, l_{m}-1\right\}$ the $l$-cycle is absent in the class of permutations corresponding to $\left\{n_{i}\right\}_{\mathcal{N}}$, while $m_{n_{i}+n_{j}}^{(\mathcal{N}-1)}$ in the denominator is the multiplicity of $n_{i}+n_{j}$ (the sum of the lower indices of $\left.K_{n_{i}, n_{j}}^{(\mathcal{N})}\right)$ in the string $\left(n_{1}, ., h_{j}, ., n_{i}+n_{j}, ., n_{\mathcal{N}}\right)$ that is equal to its multiplicity in the string $\left(n_{1}, . ., n_{\mathcal{N}}\right)$ plus one. The same definition is valid for $K_{\bar{n}, n_{i}-\bar{n}}^{(\mathcal{N}+1)}$, namely

$$
K_{\bar{n}, n_{i}-\bar{n}}^{(\mathcal{N}+1)}=\frac{m_{\bar{n}}^{(\mathcal{N}+1)}\left(m_{n_{i}-\bar{n}}^{(\mathcal{N}+1)}-\delta_{\bar{n}, n_{i}-\bar{n}}\right)}{2 m_{n_{i}}^{(\mathcal{N})}},
$$

where $m_{\bar{n}}^{(\mathcal{N}+1)}$ and $m_{n_{i}-\bar{n}}^{(\mathcal{N}+1)}$ are the the multiplicities of the values $\bar{n}$ and $n_{i}-\bar{n}$ respectively in the string $\left(n_{1}, . ., n_{i}-\bar{n}, ., n_{\mathcal{N}}, \bar{n}\right)$ and $m_{n_{i}}^{(\mathcal{N})}$ in the denominator is the multiplicity of the value $n_{i}$ (the sum of the lower indices of $K_{\bar{n}, n_{i}-\bar{n}}^{(\mathcal{N}+1)}$ ) in the string $\left(n_{1}, . ., \not h_{i}+\not h, ., n_{i}, ., n_{\mathcal{N}}\right.$ ) that is the original string $\left(n_{1}, . ., n_{\mathcal{N}}\right)$.

Eq. (26) is equivalent to Eq. (27) since the first describes the scaling behavior of the operators while the second of the couplings. To show how Eq. (27) can be derived from Eq. (26) let us consider the equations

$$
\begin{aligned}
& \left\langle\operatorname{Tr}^{\prime} Q^{n_{i}+n_{j}} \prod_{k \neq i, j}^{\mathcal{N}} \operatorname{Tr}^{\prime} Q^{n_{k}}\right\rangle=[\ldots]-L\left(n_{i}+n_{j}\right) \prod_{i=1}^{\mathcal{N}} \operatorname{Tr}^{\prime} Q^{n_{i}}, \\
& \left\langle\operatorname{Tr}^{\prime} Q^{n_{i}-\bar{n}} \operatorname{Tr}^{\prime} Q^{\bar{n}} \prod_{j \neq i}^{\mathcal{N}} \operatorname{Tr}^{\prime} Q^{n_{j}}\right\rangle=[\ldots]-4 L \bar{n}\left(n_{i}-\bar{n}\right) \prod_{i=1}^{\mathcal{N}} \operatorname{Tr}^{\prime} Q^{n_{i}},
\end{aligned}
$$

obtained directly applying Eq. (26). From these equations it is easy to see how the two couplings $\lambda_{\left(n_{1}, h_{j},, n_{i}+n_{j},, n_{\mathcal{N}}\right)}^{(\mathcal{N}-1)}$ and $\lambda_{\left(n_{1},, n_{j},, n_{i}-\bar{n},, n_{\mathcal{N}}, \bar{n}\right)}^{(\mathcal{N})+1)}$, corresponding to the two operators above, appear in the $\beta$-function for $\lambda_{\left(n_{1},, n_{\mathcal{N}}\right)}^{(\mathcal{N})}$, the coupling of $\prod_{i}^{\mathcal{N}} \operatorname{Tr}^{\prime} Q^{n_{i}}$.

The factors $K_{n_{i}, n_{j}}^{(\mathcal{N})}$ take care of the symmetry of the couplings with respect to any transpositions of the indices. The numerator in Eq. (28) is given by the number of different pairs $\left(n_{i}, n_{j}\right)$ one can couple starting from $m_{n_{i}}^{(\mathcal{N})}$ objects of type $n_{i}$ and $m_{n_{j}}^{(\mathcal{N})}$ objects of type $n_{j}$. If $n_{i} \neq n_{j}$, the number of pairs is $m_{n_{i}}^{(\mathcal{N})} m_{n_{j}}^{(\mathcal{N})}$ while, if $n_{i}=n_{j}$, the number of pairs is given by $\left(\begin{array}{c}m_{n_{i}}^{(\mathcal{N})} \\ 2\end{array}\right)=\frac{m_{n_{i}}^{(\mathcal{N})} !}{2 !\left(m_{n_{i}}^{(\mathcal{N})}-2\right) !}=\frac{1}{2} m_{n_{i}}^{(\mathcal{N})}\left(m_{n_{i}}^{(\mathcal{N})}-1\right)$. The denominator in Eq. (28) is $m_{n_{i}+n_{j}}^{(\mathcal{N}-1)}$ for $n_{i}=n_{j}$ while $2 m_{n_{i}+n_{j}}^{(\mathcal{N}-1)}$ for $n_{i} \neq n_{j}$, the factor 2 comes from exchanging $n_{i} \leftrightarrow n_{j}$.

By this procedure we can write down the one loop RG equations of the couplings of a generic product of traces of powers of the field $Q$. Now if we were interested to find one loop scaling operators it would be enough to find the real solutions of the $p_{n}-1$ independent 
equations, algebraic through Eq. (27), among the equations

$$
\lambda_{\left(n_{1}^{\prime}, \ldots, n_{\mathcal{N}^{\prime}}^{\prime}\right)}^{\left(\mathcal{N}^{\prime}\right)} \beta_{\lambda_{\left(n_{1}, \ldots, n_{\mathcal{N}}\right)}^{(\mathcal{N})}}=\lambda_{\left(n_{1}, \ldots, n_{\mathcal{N}}\right)}^{(\mathcal{N})} \beta_{\lambda_{\left(n_{1}^{\prime}, \ldots, n_{\mathcal{N}^{\prime}}^{\prime}\right)}^{\left(\mathcal{N}^{\prime}\right)}},
$$

with the constrain $\sum_{i}^{\mathcal{N}^{\prime}} n_{i}^{\prime}=\sum_{i}^{\mathcal{N}} n_{i}=n$ or alternatively to diagonalize the matrix $M$ constructed by the coefficients of $\lambda$ 's in the right-hand side of the equations (27) that has clearly rank $p_{n}$. Indeed, denoting with $\vec{\lambda}$ the $p_{n}$-vector formed by the couplings $\lambda_{\left\{n_{i}\right\}}^{(\mathcal{N})}$, Eq. (27) can be written in the following way

$$
\vec{\beta}_{\vec{\lambda}}=(d+g M) \vec{\lambda}
$$

Calling $\bar{\lambda}_{\left(\left\{n_{i}\right\}_{\mathcal{N}}\right)}^{(\mathcal{N})}$ the real solutions of Eqs.(30) (or alternatively the columns of the invertible matrix $T$ that diagonalizes $M$ ), the resulting $p_{n}$ operators

$$
\mathcal{O}_{i_{n}}=\sum_{\mathcal{N}=1}^{n} \sum_{\left\{n_{i}\right\}_{\mathcal{N}}} \bar{\lambda}_{\left(\left\{n_{i}\right\}_{\mathcal{N}}\right)}^{(\mathcal{N})} \prod_{i=1}^{\mathcal{N}} \operatorname{Tr}^{\prime} Q^{n_{i}}
$$

with $i_{n}=1, . ., p_{n}$, are one loop scaling operators with the following scaling behaviors

$$
\left\langle\mathcal{O}_{i_{n}}\right\rangle=\left[1+\left(n+\frac{\Gamma}{2} n^{2}+2 \sum_{i=1}^{\mathcal{N}} n_{i}\left(n_{i}-i\right)\right) L\right] \mathcal{O}_{i_{n}}
$$

where $n_{1} \geq n_{2} \geq \ldots \geq n_{\mathcal{N}}$ is a partition of $n$. The factor in front of $L$ is the $i_{n}$ th element of the diagonal matrix $T^{-1} M T$ and goes from the value $\left[\left(\frac{\Gamma}{2}+2\right) n^{2}-n\right]$, the most relevant, related to the partition $\{n\}$, to the value $\left[\left(\frac{\Gamma}{2}-1\right) n^{2}+2 n\right]$, related to the partition $\{1, \ldots, 1\}$. Eq. (33) is exactly what we can find also using the Young tableaux, already adopted, for instance, to evaluate the average of moments of the eigenfunctions of a particle in a random potential near the mobility edge [17].

As in that case, one-loop renormalization leads to transpositions of indices of the matrix field under the sum of upper indices, taking advantage of the charge conjugation condition (51) (see, for instance, Eqs. (16][18) $)$. Indeed the factors $\left[n+2 \sum_{i}^{\mathcal{N}} n_{i}\left(n_{i}-i\right)\right]$ are the eigenvalues of the operator $\sum_{i>j}(i j)$ which is the sum of all the transpositions $(i j)$ acting on a set of $n$ pairs $\{(1,2),(3,4), . .,(2 n-1,2 n)\}$, the indices of $n Q$-matrices, and where $(i j)$ is an identity on each pair. The dimension of the space spanned by this operator is $(2 n-1)$ !! which is equal to the sum of the dimensions of some irreducible representations of the symmetric group $S_{2 n}$, those related only to the even partitions of $2 n$ [17]. The number of even partitions of $2 n$ is also given by $p_{n}$, the number of all the partitions of $n$. For instance, 
if $n=2$ the dimension of the space is 3, (the tree vectors $v_{i}$ in Eq. (19)) but the eigenvalues are two (see Eqs. (20, 21), $v_{2}$ and $v_{3}$ are degenerate). Indeed the irreducible representation related to the partition $\{4\}$ has one dimension while the one related to the partition $\{2,2\}$ is a two-dimensional representation [17]. The additional term, $\frac{\Gamma}{2} n^{2}$, results from the sum of two contributions, the first coming from the mean values $\frac{1}{2}\left\langle\widetilde{U}^{\dagger} W W U\right\rangle$, in all the terms $\langle Q\rangle$, which give $\frac{\Gamma}{2} n$ and the second coming from $\left\langle\widetilde{U}^{\dagger} W U \ldots \widetilde{U}^{\dagger} W U\right\rangle$, appearing in in all the average values $\langle Q Q\rangle$, which give $\frac{\Gamma}{2} n(n-1)$.

Finally we can rewrite $P_{n}$ of Eq. (25) in terms of such scaling operators

$$
P_{n}=\sum_{i_{n}=1}^{p_{n}} a_{i_{n}} \mathcal{O}_{i_{n}} .
$$

For the sake of clarity we refer to an explicit example in Appendix B.

\section{RG SOLUTIONS}

As we have seen before, we have decoupled all the moments of $Q$ writing them in terms of the scaling operators $\mathcal{O}_{i_{n}}$, with $i_{n}=1, \ldots, p_{n}$, which are coupled only to the equations of $g$, the resistivity, and $\Gamma$. In terms of the couplings $a_{i_{n}}$ of the operators $\mathcal{O}_{i_{n}}$, the whole set of one-loop RG equations in $\epsilon=d-2$ expansion $\forall n$ is the following

$$
\begin{aligned}
& \beta_{g}=-\epsilon g \\
& \beta_{\Gamma}=4 g \\
& \beta_{a_{i_{n}}}=d a_{i_{n}}+\left(\mathcal{A}_{i_{n}}+\mathcal{B}_{n} \Gamma\right) g a_{i_{n}},
\end{aligned}
$$

where the coefficients $\mathcal{A}_{i_{n}}$ and $\mathcal{B}_{n}$ are defined in the following way

$$
\begin{aligned}
& \mathcal{A}_{i_{n}}=n+2 \sum_{i=1}^{\mathcal{N}} n_{i}\left(n_{i}-i\right), \quad \text { with } n_{1} \geq \ldots \geq n_{\mathcal{N}} \\
& \mathcal{B}_{n}=n^{2} / 2
\end{aligned}
$$

These decoupled RG equations can be solved easily obtaining the following solution

$$
\begin{aligned}
& g=g_{0} s^{-\epsilon} \\
& \Gamma=\Gamma_{0}+\frac{4 g_{0}}{\epsilon}\left(1-s^{-\epsilon}\right) \\
& \ln \left[\frac{a_{i_{n}}}{a_{i_{n}}}\right]=d \ln s+\frac{1}{\epsilon^{2}}\left[g_{0} s^{-2 \epsilon}\left(s^{\epsilon}-1\right)\left(\mathcal{A}_{i_{n}} \epsilon s^{\epsilon}+\mathcal{B}_{n}\left(\epsilon \Gamma_{0} s^{\epsilon}+2 g_{0}\left(s^{\epsilon}-1\right)\right)\right)\right] .
\end{aligned}
$$


In 2-dimensions the solution of the RG equations is the limit $\epsilon \rightarrow 0$ of Eqs. (40,42),

$$
\begin{aligned}
& g=g_{0}, \\
& \Gamma=\Gamma_{0}+4 g_{0} \ln s \\
& \ln \left[\frac{a_{i_{n}}}{a_{i_{n} 0}}\right]=\left(2+\mathcal{A}_{i_{n}} g_{0}+\mathcal{B}_{n} g_{0} \Gamma_{0}\right) \ln s+2 \mathcal{B}_{n} g_{0}^{2}(\ln s)^{2} .
\end{aligned}
$$

The flow equations for the original couplings in Eq. (25), called here $\lambda_{i_{n}}$ with $i_{n}=1, \ldots, p_{n}$ for simplicity, are

$$
\lambda_{i_{n}}(s)=\sum_{j_{n}} T_{i_{n} j_{n}} a_{j_{n}}=s^{B_{n} \ln s} \sum_{j_{n}, l_{n}} T_{i_{n} j_{n}} s^{A_{j_{n}}} T_{j_{n} l_{n}}^{-1} \lambda_{l_{n}},
$$

where $T$ is the invertible matrix that diagonalizes $M$ and

$$
\begin{aligned}
& A_{i_{n}}=2+\mathcal{A}_{i_{n}} g_{0}+\mathcal{B}_{n} g_{0} \Gamma_{0}, \\
& B_{n}=2 \mathcal{B}_{n} g_{0}^{2} .
\end{aligned}
$$

Considering, in the limit $s \rightarrow \infty$, only the first most relevant exponent, denoted by $A_{r_{n}}$, for which $a_{r_{n} 0} \neq 0$, we have simply

$$
\lambda_{i_{n}}(s) \simeq s^{A_{r_{n}}+B_{n} \ln s} T_{i_{n} r_{n}} \sum_{l_{n}} T_{r_{n} l_{n}}^{-1} \lambda_{l_{n}}
$$

and if $\lambda_{l_{n}}=\lambda_{i_{n}} \delta_{i_{n}, l_{n}}$ we can write

$$
\ln \left[\frac{\lambda_{i_{n}}(s)}{\lambda_{i_{n}}}\right] \simeq \ln \left[\frac{a_{r_{n}}}{a_{r_{n} 0}}\right] .
$$

Inverting Eq. (49) we find that the couplings $\lambda_{i_{n}}(s)$ reach an upper value $\Lambda$ at the length scale

$$
s=\exp \left[\frac{1}{2 B_{n}} \sqrt{A_{r_{n}}-4 B_{n} \ln \left(\frac{\Lambda}{T_{i_{n} r_{n}} \sum_{l_{n}} T_{r_{n} l_{n}}^{-1} \lambda_{l_{n}}}\right)}\right] .
$$

Notice that in general terms the flow to strong coupling regime can be tuned and slowed choosing some particular starting configurations of the couplings, namely when $\sum_{l_{n}} T_{r_{n} l_{n}}^{-1} \lambda_{l_{n}}=0$ for some $r_{n}$ related to the most relevant scaling operators.

\section{MULTIFRACTALITY AND ON-SITE PERTURBATION}

\section{A. Density of states near the band center}

An important application of the analysis done in the previous sections is in the following. By expanding in $E$ the action (2), composite operators like $E^{n} \operatorname{Tr}\left(Q^{n}\right)$ appear in the model. 
These operators can be written as linear combinations of scaling operators and among them, for each $n$, the most relevant one has dimension

$$
z_{n}=2+g\left(\frac{\Gamma}{2}+2\right) n^{2}-g n .
$$

Considering only the operator with this dimension as the most representative for $E^{n}$, in the limit $s \rightarrow \infty$, using Eq. (50), we can write

$$
\ln \left[\frac{E^{n}(s)}{E^{n}}\right] \simeq \int_{0}^{\ln s} z_{n} d \ln s^{\prime} .
$$

Let us now define the following distribution function [19]

$$
\mathcal{P}(\mathcal{Y}, s) \sim \frac{1}{\mathcal{Y} \ln s} s^{f(\ln \mathcal{Y} / \ln s)},
$$

where the function $f(\alpha)$ is defined by

$$
f(\alpha)=2-(\alpha+g)^{2} /(2 g(\Gamma+4))
$$

linked to $z_{n}$ by a Legendre transform [20]. Indeed finding $\alpha$ as solution of the following equation

$$
f^{\prime}(\alpha)+n=0
$$

we obtain

$$
\alpha(n)=n g(\Gamma+4)-g
$$

This implies that $z_{n}$ and $f(\alpha)$ are related by the following Legendre transform

$$
z_{n}=n \alpha(n)+f(\alpha(n))
$$

Now we define through the distribution function (54) the following mean value

$$
\left\langle\mathcal{Y}^{n}\right\rangle_{\mathcal{P}(\mathcal{Y}, s)} \equiv \int d \mathcal{Y} \mathcal{P}(\mathcal{Y}, s) \mathcal{Y}^{n}
$$

Changing the integral variable by $\alpha=\ln \mathcal{Y} / \ln s$, Eq. (59) becomes

$$
\left\langle\mathcal{Y}^{n}\right\rangle_{\mathcal{P}(\mathcal{Y}, s)} \sim \int d \alpha s^{n \alpha+f(\alpha)} \simeq s^{z_{n}}
$$

where we have evaluated the integral by the saddle-point method, being (57) the saddle point. Using this result we can write $E^{n}(s)$ in terms of the average value of $\mathcal{Y}^{n}$

$$
\frac{E^{n}(s)}{E^{n}} \simeq \exp \left[\int_{0}^{\ln s} z_{n} d \ln s^{\prime}\right] \sim \exp \left[\int_{0}^{\ln s} \ln \left\langle\mathcal{Y}^{n}\right\rangle_{\mathcal{P}\left(\mathcal{Y}, s^{\prime}\right)} \frac{d \ln s^{\prime}}{\ln s^{\prime}}\right]
$$


In some conditions this quantity is dominated by the tails of the distribution, namely it is determined by rare events and, therefore, can not represent the true energy scaling. From Eq. (60) we notice, indeed, that for large $n$ the tails of the distribution affect strongly the mean value of $\mathcal{Y}^{n}$. The so called typical mean values, on the contrary, give insight on the bulk of the distribution function. For this reason we will consider the following quantity

$$
\frac{E_{t y p}^{n}(s)}{E_{t y p}^{n}} \simeq \exp \left[\int_{0}^{\ln s} z_{n}^{t y p} d \ln s^{\prime}\right] \sim \exp \left[\int_{0}^{\ln s} \ln \mathcal{Y}_{t y p}^{n} \frac{d \ln s^{\prime}}{\ln s^{\prime}}\right]
$$

where now $z_{n}^{t y p}$ is the typical dimension to be determined and $\left\langle\mathcal{Y}^{n}\right\rangle$ is replaced by $\mathcal{Y}_{t y p}^{n}=$ $\exp \left\langle\ln \mathcal{Y}^{n}\right\rangle$ with

$$
\left\langle\ln \mathcal{Y}^{n}\right\rangle_{\mathcal{P}(\mathcal{Y}, s)}=\int d \mathcal{Y} \mathcal{P}(\mathcal{Y}, s) \ln \mathcal{Y}^{n} \sim n \ln s \int d \alpha \alpha s^{f(\alpha)},
$$

where the integral can not be extended to $-\infty$ otherwise we would obtain irrelevant operators. However from Eq. (63) we can see immediately that the regions of $\alpha$ where $f(\alpha)<0$ give negligible contributions to the typical value $\frac{E_{t y p}^{n}(s)}{E_{t y p}^{n}}$. Let us consider the solution of $f(\alpha)=0$

$$
\bar{\alpha}=2 \sqrt{g(\Gamma+4)}-g=n_{c} g(\Gamma+4)-g,
$$

discarding the other solution that is irrelevant in all regimes of disorder. In Eq. (64) we have introduced the factor

$$
n_{c}=\frac{2}{\sqrt{g(\Gamma+4)}}
$$

in order to write $\bar{\alpha}$ in analogy with Eq. (57). A more convenient way to remove from consideration all the rare events with large $|\alpha|$ but with very small weight $s^{f(\alpha)}$ with $f(\alpha)<0$ is to write $\mathcal{Y}_{t y p}^{n}$ in the same form of Eq. (60) but with a restriction in the integration range

$$
\mathcal{Y}_{t y p}^{n} \sim \int_{f(\alpha) \geq 0} d \alpha s^{n \alpha+f(\alpha)}
$$

since in the region of $f(\alpha) \geq 0$ we can expand $s^{n \alpha} \simeq 1+n \alpha \ln s$. The same definition of typical values expressed by Eq. (66) has been already used in Ref. [19] for the typical inverse participation ratios.

We find that for $n>n_{c}$ the saddle-point (57) is outside the integration domain since $f(\alpha)<0$. The main contribution in the integral (66) is then due to the boundary $\bar{\alpha}$, that do not depend on $n$, implying

$$
z_{n}^{t y p}=n \bar{\alpha}=n(2 \sqrt{g(\Gamma+4)}-g) .
$$


For $n<n_{c}$ instead the integral is determined again by the saddle-point (57) since $\alpha$ is inside the integration domain being $f(\alpha)>0$. In this case we obtain $z_{n}^{t y p}=z_{n}$.

Summarizing we have the following typical dimension

$$
z_{n}^{\text {typ }}= \begin{cases}2+g\left(\frac{\Gamma}{2}+2\right) n^{2}-g n, & \text { for } n<\frac{2}{\sqrt{g(\Gamma+4)}} \\ 2 n \sqrt{g(\Gamma+4)}-g n, & \text { for } n \geq \frac{2}{\sqrt{g(\Gamma+4)}}\end{cases}
$$

The so called dynamical exponent [12] defined by

$$
z(s)=z_{n}^{t y p} / n
$$

in the strong coupling regime, where $\Gamma \geq \frac{4(1-g)}{g}$, has therefore the following scaling behavior for all value of $n$

$$
z(s) \simeq 2 \sqrt{g \Gamma} \simeq 4 g_{0} \sqrt{\ln s}
$$

Calling $\Lambda_{t y p}=\sqrt[n]{E_{t y p}^{n}(s)}$ the upper energy cut-off and $C=\frac{8 g_{0}}{3}$ a positive constant, we have finally

$$
\frac{\Lambda_{t y p}}{E_{t y p}} \simeq \exp \left[\int_{0}^{\ln s} z\left(s^{\prime}\right) d \ln s^{\prime}\right]=\exp \left[C(\ln s)^{\frac{3}{2}}\right] .
$$

Now we can easily calculate the density of states $\rho$ from its scaling equation [8]

$$
\frac{d \rho}{d \ln s^{\prime}}=\left[z\left(s^{\prime}\right)-2\right] \rho
$$

Integrating over the scaling factor up to $s \equiv s\left(\Lambda_{t y p}\right)$, we obtain

$$
\ln \frac{\rho(s)}{\rho_{0}}=C(\ln s)^{\frac{3}{2}}-2 \ln s .
$$

From Eq. (70) we have

$$
s=\exp \left[\frac{1}{C} \ln \left(\frac{\Lambda_{t y p}}{E_{t y p}}\right)\right]^{\frac{2}{3}},
$$

obtaining for the density of states the following behavior in energy

$$
\rho\left(E_{t y p}\right)=\rho_{0} \frac{\Lambda_{t y p}}{E_{t y p}} \exp \left\{-2\left[\frac{1}{C} \ln \left(\frac{\Lambda_{t y p}}{E_{t y p}}\right)\right]^{\frac{2}{3}}\right\} .
$$

We find that the density of states shows a weaker divergence than that obtained by Gade and Wegner [4, 5] who found the exponent $\frac{1}{2}$ on the logarithm. The final result (74), on the contrary, is in perfect agreement with the density of states predicted in Refs. 12, 13, 14]. 


\section{B. On-site disorder}

In the presence of small on-site disorder or same-sublattice regular hopping, terms like $c^{n} \operatorname{Tr} Q^{2 n}$ appear in the theory [8], responsible for chiral symmetry breaking of the two sublattice models. Following the same steps described previously, we have, in this case,

$$
z_{n}=2+4 g\left(\frac{\Gamma}{2}+2\right) n^{2}-2 g n
$$

related to the function

$$
f(\alpha)=2-(\alpha+2 g)^{2} /(8 g(\Gamma+4)),
$$

through the following value of $\alpha$

$$
\alpha(n)=4 n g(\Gamma+4)-2 g
$$

by the Legendre transform in Eq. (58). Defining again the typical value for $c^{n}$, we get

$$
z_{\text {typ }}=n(4 \sqrt{g(\Gamma+4)}-2 g)
$$

meaning that the dynamic exponent is

$$
z(s) \simeq 4 \sqrt{g \Gamma} \simeq 8 g_{0} \sqrt{\ln s}
$$

and

$$
\frac{\Lambda_{t y p}}{c_{t y p}} \simeq \exp \left[\frac{16}{3 g_{0}}(\ln s)^{\frac{3}{2}}\right]=\exp \left[2 C(\ln s)^{\frac{3}{2}}\right] .
$$

The coefficient $2 C$ in Eq. (180) is twice the value which appears in Eq. (170). For this reason, considering the two on-site perturbations due either to a finite potential energy $E$ or to an on-site disorder with strength $c$, if $E \sim c$, the crossover from the chiral symmetry to the standard one occurs first in the presence of the latter source of symmetry breaking.

\section{CONCLUSIONS}

In this paper we have computed the anomalous scaling dimensions of an infinite family of operators in a non-linear $\sigma$-model induced under RG by an on-site perturbation. We have applied this analysis to calculate the density of states near the chemical potential.

The new result of the present work is to prove that the same expression for the density of states, already obtained through other approaches [13, 14] which take advantage of the RG

method proposed by Carpentier and Le Doussal [21], can be derived also within the more conventional non-linear $\sigma$-model approach based on the replica method. 


\section{Acknowledgments}

The author wishes to thank Michele Fabrizio for helpful comments and discussions.

\section{APPENDIX A}

In this appendix we present a more general version of Eq. (26). Defining the operators

$$
\begin{array}{ll}
\theta_{m, n}^{(i)}=A_{m} Q A_{m+1} Q \ldots \ldots A_{n-1} Q A_{n} Q, & \text { for } m<n, \\
\theta_{m, n}^{(i)}=A_{m} Q A_{m+1} Q \ldots . A_{n_{i}-1} Q A_{n_{i}} Q A_{1} Q \ldots . A_{n-1} Q A_{n} Q, & \text { for } m>n,
\end{array}
$$

where $A_{n}$ are some symmetric or antisymmetric matrices in replica and frequency spaces, we obtain the following average value over fast modes

$$
\begin{aligned}
& \left\langle\prod_{i=1}^{\mathcal{N}} \operatorname{Tr}^{\prime} \theta_{1, n_{i}}^{(i)}\right\rangle=\left[1+L\left(2 \sum_{i=1}^{\mathcal{N}} n_{S_{i}}+n+n^{2} \frac{\Gamma}{2}\right)\right] \prod_{i=1}^{\mathcal{N}} \operatorname{Tr}^{\prime} \theta_{1, n_{i}}^{(i)} \\
& -L\left[4 \sum_{i>j} \prod_{k \neq i, j}^{\mathcal{N}} \operatorname{Tr}^{\prime} \theta_{1, n_{k}}^{(k)} \sum_{l_{i}=1}^{n_{i}} \sum_{l_{j}=1}^{n_{j}} \operatorname{Tr}^{\prime}\left(\theta_{l_{i}+1, l_{i}}^{(i)} \theta_{l_{j}, l_{j}-1}^{(j)}\right)\right. \\
& \left.+2 \sum_{i=1}^{\mathcal{N}}\left(\prod_{j \neq i}^{\mathcal{N}} \operatorname{Tr}^{\prime} \theta_{1, n_{j}}^{(j)} \sum_{p=1}^{n_{i}-1} \sum_{l=p}^{n_{i}-1} \operatorname{Tr}^{\prime} \theta_{p, n_{i}-l}^{(i)} \operatorname{Tr}^{\prime} \theta_{n_{i}-l+1, p-1}^{(i)}\right)\right],
\end{aligned}
$$

where, as before, $n=\sum_{i}^{\mathcal{N}} n_{i}$ and the numbers $n_{S_{i}}$ are defined by

$$
n_{S_{i}}=\sum_{m=1}^{n_{i}-1} \sum_{k=1}^{m} \prod_{j=k+1}^{n_{i}} S\left(A_{j}\right)
$$

in which $S\left(A_{j}\right)$ is a sign, +1 if $A_{j}$ is a symmetric operator and -1 if $A_{j}$ antisymmetric. If all $A_{j}$ are symmetric and equal, namely $A_{j}=A=A^{t} \forall j$, as in the particular case described by Eq. (26), where $A$ is the identity in all the spaces, one has simply $n_{S_{i}}=\frac{1}{2} n_{i}\left(n_{i}-1\right)$.

\section{APPENDIX B}

Here we consider, as an example, the case with $n=4$. The polynomial in Eq. (25) then is the following

$P_{4}=\left[\lambda_{(4)}^{(1)} \operatorname{Tr}^{\prime} Q^{4}+\lambda_{(3,1)}^{(2)} \operatorname{Tr}^{\prime} Q^{3} \operatorname{Tr}^{\prime} Q+\lambda_{(2,2)}^{(2)}\left(\operatorname{Tr}^{\prime} Q^{2}\right)^{2}+\lambda_{(2,1,1)}^{(3)} \operatorname{Tr}^{\prime} Q^{2}\left(\operatorname{Tr}^{\prime} Q\right)^{2}+\lambda_{(1,1,1,1)}^{(4)}\left(\operatorname{Tr}^{\prime} Q\right)^{4}\right]$ 
and we have from Eqs. (27, 29) the following $\beta$-functions

$$
\begin{aligned}
& \beta_{\lambda_{(4)}^{(1)}}=d \lambda_{(4)}^{(1)}+g\left[(16+8 \Gamma) \lambda_{(4)}^{(1)}-12 \lambda_{(3,1)}^{(2)}-16 \lambda_{(2,2)}^{(2)}\right], \\
& \beta_{\lambda_{(3,1)}^{(2)}}=d \lambda_{(3,1)}^{(2)}+g\left[(10+8 \Gamma) \lambda_{(3,1)}^{(2)}-8 \lambda_{(4)}^{(1)}-16 \lambda_{(2,1,1)}^{(3)}\right], \\
& \beta_{\lambda_{(2,2)}^{(2)}}=d \lambda_{(2,2)}^{(2)}+g\left[(8+8 \Gamma) \lambda_{(2,2)}^{(2)}-4 \lambda_{(4)}^{(1)}-4 \lambda_{(2,1,1)}^{(3)}\right], \\
& \beta_{\lambda_{(2,1,1)}^{(3)}}=d \lambda_{(2,1,1)}^{(3)}+g\left[(6+8 \Gamma) \lambda_{(2,1,1)}^{(3)}-6 \lambda_{(3,1)}^{(2)}-4 \lambda_{(2,2)}^{(2)}-24 \lambda_{(1,1,1,1)}^{(4)}\right], \\
& \beta_{\lambda_{(1,1,1,1)}^{(4)}}=d \lambda_{(1,1,1,1)}^{(4)}+g\left[(4+8 \Gamma) \lambda_{(1,1,1,1)}^{(4)}-2 \lambda_{(2,1,1)}^{(3)}\right] .
\end{aligned}
$$

Solving the four independent equations (301) one can obtain the following real solutions $\quad\left(\bar{\lambda}_{(4)}^{(1)}, \bar{\lambda}_{(3,1)}^{(2)}, \bar{\lambda}_{(2,2)}^{(2)}, \bar{\lambda}_{(2,1,1)}^{(3)}, \bar{\lambda}_{(1,1,1,1)}^{(4)}\right)=$ $\left\{c_{1}(-48,32,12,-12,1), c_{2}(8,4,-2,-5,1), c_{3}(2,-8,7,-2,1), c_{4}(-4,-2,-2,1,1), c_{5}(6,8,3,6,1)\right\}$ with $c_{i}$ arbitrary constants. Inserting these solutions in Eq. (32) we obtain five scaling operators that behave in the following way

$$
\begin{aligned}
& \left\langle\mathcal{O}_{1}\right\rangle=(1+(28+8 \Gamma) L) \mathcal{O}_{1}, \\
& \left\langle\mathcal{O}_{2}\right\rangle=(1+(14+8 \Gamma) L) \mathcal{O}_{2}, \\
& \left\langle\mathcal{O}_{3}\right\rangle=(1+(8+8 \Gamma) L) \mathcal{O}_{3}, \\
& \left\langle\mathcal{O}_{4}\right\rangle=(1+(2+8 \Gamma) L) \mathcal{O}_{4}, \\
& \left\langle\mathcal{O}_{5}\right\rangle=(1+(-8+8 \Gamma) L) \mathcal{O}_{5}
\end{aligned}
$$

For all of them Eq. (33) is verified: $\mathcal{O}_{1}$ is related to the partition $\{4\}, \mathcal{O}_{2}$ to $\{3,1\}, \mathcal{O}_{3}$ to $\{2,2\}, \mathcal{O}_{4}$ to $\{2,1,1\}$ and finally $\mathcal{O}_{5}$ is related to the partition $\{1,1,1,1\}$.

Alternatively from the $\beta$-functions written above we can construct the matrix $M$ which appears in Eq. (31)

$$
M=\left(\begin{array}{rrrrr}
16 & -12 & -16 & 0 & 0 \\
-8 & 10 & 0 & -16 & 0 \\
-4 & 0 & 8 & -4 & 0 \\
0 & -6 & -4 & 6 & -24 \\
0 & 0 & 0 & -2 & 4
\end{array}\right)+8 \Gamma \mathbb{I},
$$


where $\mathbb{I}$ is the $5 \times 5$ identity matrix. $M$ is diagonalized by the invertible matrix

$$
T=\left(\begin{array}{rrrrr}
-48 & 8 & 2 & 4 & 6 \\
32 & 4 & -8 & -2 & 8 \\
12 & -2 & 7 & -2 & 3 \\
-12 & -5 & -2 & 1 & 6 \\
1 & 1 & 1 & 1 & 1
\end{array}\right)
$$

and its diagonal form is

$$
T^{-1} M T=\operatorname{diag}\{28,14,8,2,-8\}+8 \Gamma \mathbb{I} .
$$

[1] G. Theodorou and M.H. Cohen, Phys. Rev. B 13, 4593 (1976).

[2] T.P. Eggarter and R. Riedinger, Phys. Rev. B 18, 569 (1978).

[3] F.J. Wegner, Phys. Rev. B 19, 783 (1979).

[4] R. Gade and F. Wegner, Nucl. Phys. B 360, 213 (1991).

[5] R. Gade, Nucl. Phys. B 398, 499 (1993).

[6] L. Balents and M.P.A. Fisher, Phys. Rev. B 56, 12970 (1997).

[7] M. Steiner, M. Fabrizio, and A. O. Gogolin, Phys. Rev. B 57, 8290 (1998).

[8] M. Fabrizio and C. Castellani, Nucl. Phys. B 583, 542 (2000).

[9] S. Guruswamy, A. LeClair, and A.W.W. Ludwig, Nucl. Phys. B 583, 475 (2000).

[10] C. Mudry, P. W. Brouwer and A. Furusaki, Phys. Rev. B 63, 129901 (2001).

[11] S. Ryu, C. Mudry and A. Furusaki, Phys. Rev. B 70, 195329 (2004).

[12] O. Motrunich, K. Damle, and D.A. Huse, Phys. Rev. B 65, 064206 (2001).

[13] C. Mudry, S. Ryu, and A. Furusaki, Phys. Rev. B 67, 064202 (2003).

[14] H. Yamada and T. Fukui, Nucl. Phys. B 679, 632 (2004).

[15] K.B. Efetov, A.I.Larkin, and D.E. Khmel'nitsky, Zh. Eksp. Teor. Fiz. 79, 1120 (1979) [Sov. Phys. JETP 52, 568 (1980)].

[16] G. Wilson and J. Kogut, Phys. Rep. 12, 77 (1974); A.M. Polyakov, Phys. Lett. B 59, 79 (1975).

[17] F.J. Wegner, Z. Phys. B 36, 209 (1980).

[18] F.J. Wegner, Z. Phys. B 35, 207 (1979). 
[19] A.D. Mirlin and F. Evers, Phys. Rev. B 62, 7920 (2000).

[20] G. Paladin and A. Vulpiani, Phys. Rep. 156, 147 (1987).

[21] D. Carpentier and P. Le Doussal, Nucl. Phys. B 558, 565 (2000). 